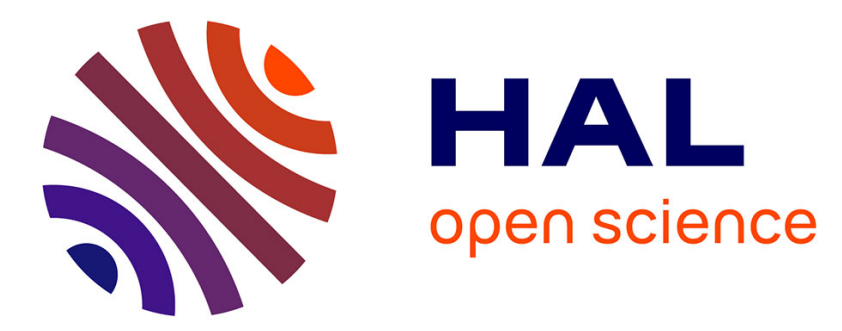

\title{
Prompt Photon Identification in the ALICE Experiment: The Isolation Cut Method
}

G. Conesa, H. Delagrange, J. Diaz, Y.V. Kharlov, Y. Schutz

\section{To cite this version:}

G. Conesa, H. Delagrange, J. Diaz, Y.V. Kharlov, Y. Schutz. Prompt Photon Identification in the ALICE Experiment: The Isolation Cut Method. Nuclear Instruments and Methods in Physics Research Section A: Accelerators, Spectrometers, Detectors and Associated Equipment, 2007, 580, pp.14461459. 10.1016/j.nima.2007.06.014 . in2p3-00138058

\section{HAL Id: in2p3-00138058 https://hal.in2p3.fr/in2p3-00138058}

Submitted on 24 Mar 2007

HAL is a multi-disciplinary open access archive for the deposit and dissemination of scientific research documents, whether they are published or not. The documents may come from teaching and research institutions in France or abroad, or from public or private research centers.
L'archive ouverte pluridisciplinaire HAL, est destinée au dépôt et à la diffusion de documents scientifiques de niveau recherche, publiés ou non, émanant des établissements d'enseignement et de recherche français ou étrangers, des laboratoires publics ou privés. 


\title{
Prompt photon identification in the ALICE experiment:The Isolation Cut Method
}

\author{
G. Conesa ${ }^{\mathrm{a}, \mathrm{b}, *}$ H. Delagrange ${ }^{\mathrm{b}}$ J. Díaz ${ }^{\mathrm{a}}$ Y.V. Kharlov ${ }^{\mathrm{c}}$ Y. Schutz ${ }^{\mathrm{b}, \mathrm{d}}$

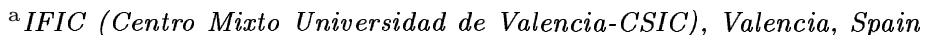 \\ ${ }^{\mathrm{b}}$ SUBATECH (Ecole des Mines, Université de Nantes, CNRS/IN2P3), Nantes, France \\ ${ }^{\mathrm{C}}$ Institute for High-Energy Physics, Protvino, Russia \\ ${ }^{\mathrm{d}}$ CERN, Genève, Switzerland
}

\begin{abstract}
The ALICE experiment at LHC will detect and identify prompt photons and light neutral mesons with the PHOS and EMCal detectors. Charged particles will be detected and identified by the central tracking system. In this paper, a method to identify prompt photons and to separate them from the background of hadrons and decay photons in PHOS with the help of isolation cuts is presented.
\end{abstract}

Key words: High-energy gamma rays, electromagnetic calorimeters, PWO scintillators, quark-gluon plasma. PACS: 25.75.Nq, 24.10.Lx, 25.75.-q, 29.40.Vj

\section{Introduction}

The experimental study of hadron jets at LHC is expected to provide decisive data for understanding the properties of the quark gluon plasma (QGP) formed in ultra-relativistic nucleus-nucleus collisions [1]. Hadron jets are generated by the hadronization of final-state partons with high transverse momentum $\left(p_{T}\right)$ scattered in primary collisions. Bjorken suggested more than 20 years ago that partons propagating through a nuclear medium are affected by an energy loss which is strongly dependent on the color charge density of the medium [2]. The phase transition of the

\footnotetext{
* Corresponding author

Email address: Gustavo.Conesa. Balbastre@cern.ch (G. Conesa).
}

medium should manifest as a modification of the energy spectrum of jet hadrons known as the jet quenching effect. This effect has indeed been observed in central Au-Au collisions at $\sqrt{s_{N N}}=130$ and $200 \mathrm{GeV}$ in measurements of high $p_{T}$ charged and neutral hadrons $\left(p_{T} \sim 2-15 \mathrm{GeV} / c\right)$ by various RHIC experiments [3-10] in which the yields of inclusive charged hadrons and $\pi^{\circ}$ mesons are found to be suppressed by as much as a factor 5 , independently of their $p_{T}$ value, compared to the properly scaled $p p, d-\mathrm{Au}$ and peripheral $\mathrm{Au}-\mathrm{Au}$ yields.

The ALICE experiment will extend such studies up to $\sqrt{s_{N N}}=5.5 \mathrm{TeV}$. Due to high cross sections of hard processes at LHC energies, jets will be abundantly produced in ALICE $\left(10^{5}\right.$ jets with $p_{T}>100 \mathrm{GeV} / c$ per year) enabling both inclusive and exclusive jet measurements. In particular, 
jet topology (jet shape, jet heating, fragmentation function, etc.) could be measured to obtain information about the energy redistribution of the jet after traversing the medium [11]. This kind of studies requires identification of jets and measurement, as accurately as possible, of the parton or jet energy before and after quenching. A very attractive method to perform this measurement is to tag jets with prompt photons emitted opposite to the jet direction. Photons emerge almost unaltered from the dense medium and provide a measurement of the original energy of the parton emitted in the opposite direction. On one hand, this coincidence technique will help to localize spatially the jet and on the other hand it allows to build the parton fragmentation function without the need of reconstructing the jet energy from the detected hadrons. Thus, medium effects could be characterized by a change of the shape of the fragmentation function, i. e., by a redistribution rather than a reduction of the jet energy.

In ALICE, photons are detected by the photon spectrometer PHOS [12-14] and the future electromagnetic calorimeter EMCal $[15,16]$. The identification power of prompt photons at low $p_{T}$ is limited by the background created by decay photons (mainly, $\pi^{0} \rightarrow \gamma \gamma$ ). At higher $p_{T}$, where the $\gamma / \pi^{0}$ ratio increases, the reduction of the opening angle between photons produces the merging of both decay photons into a single shower mimicking a single photon. These merged photons are a background for prompt photons particularly difficult to suppress. In this paper, we will discuss the feasibility of identifying prompt photons in $p p$ and heavy-ion collisions by shower topology analysis ${ }^{1}[13,14]$ and isolation criteria.

In a forthcoming paper, we discuss the identification of prompt-photon jet events and the reconstruction of hadron-jet features [17].

\footnotetext{
1 Particles interacting with the calorimeter will produce a particle shower. The shower will make signal in different cells of which the detector is subdivided. The shape of the clusters of cells fired by the particle informs about its identity.
}

Table 1

TPC and PHOS acceptances. The physical TPC $\eta$ acceptance is larger $(|\eta|<0.9)$, but we take this lower value to ensure a good track matching. We show here the EMCAL $\phi$ acceptance used in our analysis in Ref. [17] which is not the final acceptance, now under discussion.

\begin{tabular}{cccc} 
Detector & $|\eta|$ & $\phi_{\min }$ & $\phi_{\max }$ \\
\hline \hline PHOS & 0.12 & $220^{\circ}$ & $320^{\circ}$ \\
EMCAL & 0.7 & $60^{\circ}$ & $180^{\circ}$ \\
TPC & 0.7 & $0^{\circ}$ & $360^{\circ}$ \\
\hline
\end{tabular}

\section{Event simulation and main features of detected event reconstruction}

In ALICE, photons will be detected by the photon spectrometer PHOS which enables to measure with high precision their 4-momenta, although only with a reduced acceptance and also by the future electromagnetic calorimeter EMCal which has larger acceptance (Table 1) but lower resolution than PHOS. Charged particles streaming from jets are detected by the TPC, the main detector of the ALICE central tracking system. Here, we focus on PHOS to detect photons. The acceptances of all the detectors relevant for this study are reported in Table 1 and their location is pictured in Fig. 1. A full description of the ALICE detector can be found in Ref. [18].

Concerning event generation, we considered prompt photon production in the leading order of the Standard Model and next to leading order fragmentation photons. The aim of our investigation is to develop an algorithm capable of identifying prompt photons and of minimizing the wrong identification of particles, mainly $\pi^{0}$, as prompt photons. There are significant discrepancies between the event generators PYTHIA, HERWIG and NLO calculations. In the present state of knowledge, there is no strong argument in favor of any of these generators. In such circumstances, we assumed that PYTHIA is a reasonable option to demonstrate the validity of our approach.

In this paper, those events containing a prompt photon and a jet are referred to as $\gamma$-jet events. The dominant processes contributing at the parton level are the Compton and annihilation processes: 


$$
\begin{aligned}
& g+q \rightarrow \gamma+q, \\
& q+\bar{q} \rightarrow \gamma+g .
\end{aligned}
$$

These processes were simulated by the event generator PYTHIA $6.203[19,20]$ for $p p$ collisions at $\sqrt{s}=5.5 \mathrm{TeV}$. The default parton distribution function, GRV 94L [21], was used. Since the production cross sections drop quickly with $p_{T}$, events were sampled in the following $\hat{p_{T}}$ bins $^{2}$ (about 10,000 events per bin): [20,40], [40,60], [60,80], and $[80,100] \mathrm{GeV} / c$. To obtain a continuous differential cross sections over the whole $\hat{p_{T}}$-range, these four samples were weighted by their corresponding cross sections calculated by PYTHIA. In order to enrich the sample with events detected in PHOS, we restricted the generated prompt photons to a solid angle slightly larger than the PHOS acceptance to avoid possible boundary effects, defined by the ranges $\left|\eta_{\gamma}\right|<0.2$ for the pseudorapidity in the event center-of-mass system and $200^{\circ}<\phi_{\gamma}<$ $340^{\circ}$ for the azimuthal angle.

Events with two jets in the final state, called jetjet events, are a significant source of background for $\gamma$-jet events. They were simulated by hard QCD $2 \rightarrow 2$ processes in the leading pQCD order,

$$
\begin{gathered}
q+q \rightarrow g+g, \\
q+q^{\prime} \rightarrow q+q^{\prime}, \\
q+\bar{q} \rightarrow q+\bar{q}, \\
q+g \rightarrow q+g, \\
g+g \rightarrow g+g, \\
g+g \rightarrow q+q^{\prime} .
\end{gathered}
$$

These processes contribute to the $\gamma$-jet background through hard fragmentation photon and $\pi^{0}$-meson production as seen in Ref. [22].

To simulate a continuous $p_{T^{-}}$spectrum of $\pi^{0}$ mesons from 20 to $100 \mathrm{GeV} / c$, we generated hard QCD processes in eight bins of $10 \mathrm{GeV} / c$ width in the $\hat{p_{T}}$ range from 30 to $100 \mathrm{GeV} / c$ and in four more bins of $50 \mathrm{GeV} / c$ width in the $\hat{p_{T}}$ range from 100 to $300 \mathrm{GeV} / c$ (about 150,000 events per bin). The whole $p_{T}$ spectrum was taken as the sum of these

$\overline{2 \text { Here } \hat{p_{T}}}$ refers to the transverse momentum of the hard $2 \rightarrow 2$ processes in their center-of-mass system.

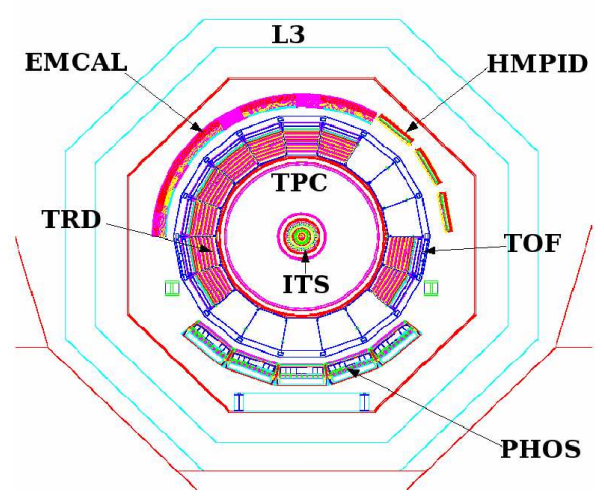

Fig. 1. Layout of various ALICE detectors.

samples weighted by their corresponding cross sections. The generation of hard QCD processes was restricted in the process center-of-mass system to $\left|y_{\text {parton }}\right|<0.2$ and in the event center-of-mass system to $\left|\eta_{\text {jet }}\right|<0.15$, without any azimuthal angle limitation. Due to the small PHOS acceptance, we had to restrict the rapidity range of generated jet-jet events more severely than that of the $\gamma$-jet events.

To simulate events corresponding to $\mathrm{Pb}-\mathrm{Pb}$ collisions, we assumed that a $\mathrm{Pb}-\mathrm{Pb}$ collision is equivalent to a $p p$ collision containing our signal, plus an underlying heavy-ion collision, constituting the background. Thus, $p p$ collisions generated by PYTHIA, were merged with heavy-ion collisions produced by the HIJING 1.36 [23] event generator for $\mathrm{Pb}-\mathrm{Pb}$ collisions at $\sqrt{s_{N N}}=5.5 \mathrm{ATeV}$ and impact parameter $b<2 \mathrm{fm}$.

\subsection{Expected experimental rates}

The $p_{T}$ distributions obtained from simulations were normalized to the number of events expected in a standard year of LHC running (see Table 2). As we simulated different $\hat{p_{T}}$ bins, each one with a different number of events, we proceeded in the following way:

- The raw distributions were normalized to the number of simulated events in each $\hat{p_{T}}$ bin and to the cross sections calculated by PYTHIA. Finally, the different distributions were combined to obtain the differential cross section as a function of $p_{T}$. 
Table 2

Beam luminosity and running time for the ALICE experiment during a standard year of running at LHC.

\begin{tabular}{ccc}
\hline Collision & $\mathcal{L}\left(\mathrm{cm}^{-2} \mathrm{~s}^{-1}\right)$ & $\mathrm{t}(\mathrm{s})$ \\
\hline \hline$p p$ & $10^{30}$ & $10^{7}$ \\
$\mathrm{~Pb}-\mathrm{Pb}$ & $0.510^{27}$ & $10^{6}$ \\
\hline
\end{tabular}

Table 3

Values of the nuclear overlap function for $\mathrm{Pb}-\mathrm{Pb}$ collisions and for different collision centralities taken from Appendix I of [24].

\begin{tabular}{ccc}
\hline Centrality (\%) & $f_{C}$ & $\left\langle T_{A A}\right\rangle_{C}\left(\mathrm{mb}^{-1}\right)$ \\
\hline \hline 5 & 0.05 & 26.0 \\
10 & 0.1 & 23.2 \\
minimum bias & 1 & 5.58 \\
\hline
\end{tabular}

- The differential cross section was multiplied by the integrated luminosity expected in one running period: the luminosity $\mathcal{L}$ and running time are listed in Table 2. The cross sections in $\mathrm{Pb}-\mathrm{Pb}$ collisions were obtained from those in $p p$ collisions by applying the "binary scaling" (Eq. (3)) described in details in Refs. [24,25]:

$$
\left(\frac{d^{2} \sigma_{A A}}{d p_{T} d y}\right)_{C}=\left\langle T_{A A}\right\rangle_{C} \cdot \sigma_{A A}^{g e o} \cdot f_{C} \cdot \frac{d^{2} \sigma_{p p}}{d p_{T} d y}
$$

where $\left\langle T_{A A}\right\rangle_{C}$ is the product of the nuclear overlap function for the corresponding centrality class $C, f_{C}$ is the the centrality factor and $\sigma_{A A}^{g e o}=7745 \mathrm{mb}$ is the geometrical cross section Ref. [24]. The corresponding values for different centralities are listed in Table 3 . The cross section was averaged on centrality to obtain the cross section for minimum bias collisions.

We obtained by the above procedure the spectra of prompt photons ( $\gamma$-jet) and $\gamma$-like (jet-jet) in $p p$ and $\mathrm{Pb}-\mathrm{Pb}$ collisions at $5.5 \mathrm{~A} \mathrm{TeV}$ expected to be detected in the PHOS acceptance during a standard year of LHC running, displayed in Fig. 2.

\subsection{Background contributions}

Background events are due mainly to decay and bremsstrahlung photons and misidentified hadrons. Decay photons from jet-jet events can be detected in PHOS as either single photons or
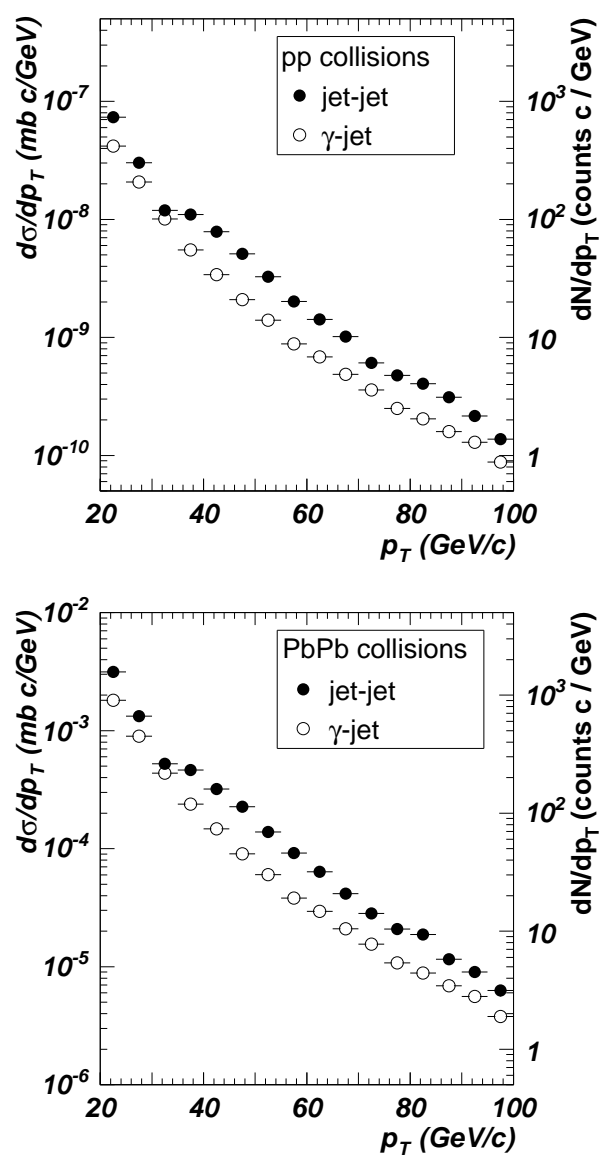

Fig. 2. Spectra of prompt photons ( $\gamma$-jet events, o) and $\gamma$-like (single and overlapped photons from jet-jet events, -) detected in PHOS, for $p p$ (up) and minimum bias $\mathrm{Pb}-\mathrm{Pb}$ (down) collisions at $\sqrt{s_{N N}}=5.5 \mathrm{~A} \mathrm{TeV}$. Differential cross sections are given on the left $y$-axis of the plots and the expected number of counts on the right $y$-axis. Both quantities are determined for a standard LHC running year.

merged clusters (also called one-cluster $\pi^{0}$ ). Due to the PHOS geometry, overlapped clusters happen essentially for $\pi^{0}$ of energy beyond $30 \mathrm{GeV}$. These photons constitute the main background for prompt photon identification and contribute significantly to the inclusive photon spectrum. The spectra of the different photon-like events and their relative contribution to the prompt-photon spectrum for $p p$ collisions are given in Fig. 3. 

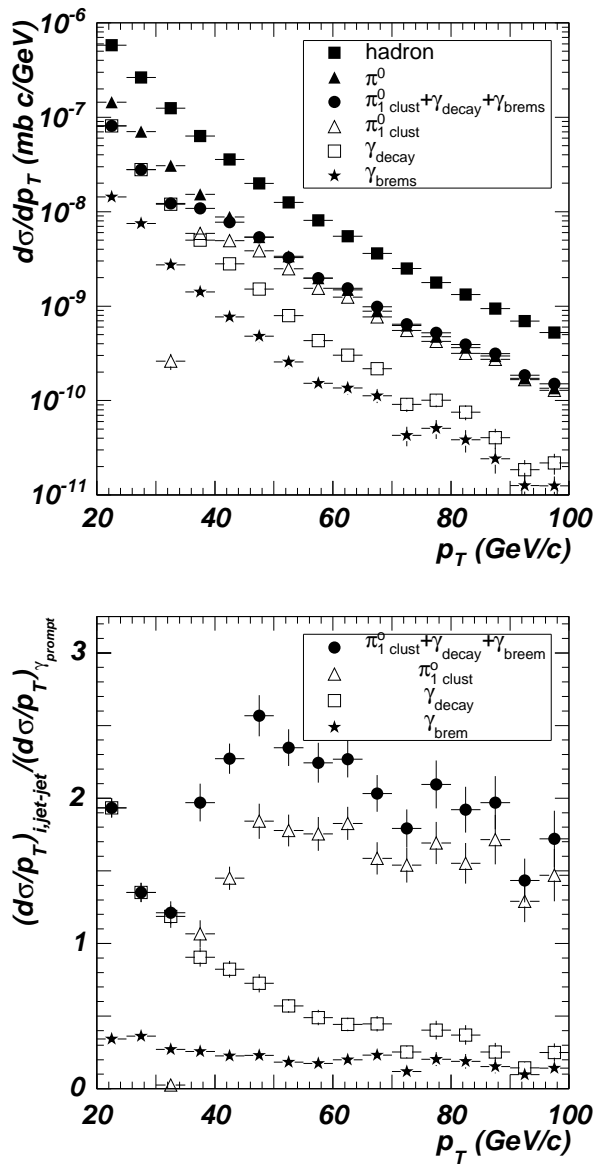

Fig. 3. Upper frame: spectra of different photon-like particles detected in PHOS streaming from a jet-jet event in $p p$ collisions at $\sqrt{s}=5.5 \mathrm{TeV} ;(\boldsymbol{\Delta})$ total hard $\pi^{0} ;(\Delta)$ onecluster $\pi^{0}$ detected in PHOS; $(\square)$ photons from decay (not overlapped); $(\bullet)$ one-cluster $\pi^{0}$ plus photons from decay and photons from bremsstrahlung; (ם) total hadron spectrum; ( $\star$ ) photons from bremsstrahlung. Lower frame: Ratio of generated photon-like particles in jet-jet events to prompt photon in $\gamma$-jet events

\section{Response of the detectors}

In this section, we describe the response functions of PHOS and the TPC. We used the simulated PYTHIA $(p p)$ and PYTHIA + HIJING $(\mathrm{Pb}-$ $\mathrm{Pb}$ ) events described in Section 2, and we performed a full-fledged Monte Carlo simulation of the transport of particles in PHOS. The whole information of the charged particles collected by the ALICE central tracking system was used, but the fast reconstruction method described in Section 3.2 was applied.

\section{1. $P H O S$}

The response of a PHOS prototype was measured at CERN in test-beam experiments and it was found that the energy resolution is given by,

$$
\frac{\Delta E}{E}=\frac{0.013}{E} \oplus \frac{0.036}{\sqrt{E}} \oplus 0.0112,
$$

where the energy is given in $\mathrm{GeV}$ units $^{3}[14,26]$. Simulation software, within the frame of AliRoot [27], was tuned to reproduce this energy resolution.

The position resolution at different incidence angles was studied by simulations. We obtained,

$$
\sigma_{x}=\frac{0.229}{\sqrt{E}} \oplus 0.096
$$

where energies are given in $\mathrm{GeV}$ and positions in $\mathrm{cm}$. For more details see $[13,14]$. The PHOS energy threshold is $0.5 \mathrm{GeV}$.

\subsection{Charged particle detector}

The fast reconstruction provides a parametrized response of ITS [28] and TPC [29] for long-lived charged particles. In this fast reconstruction [30], charged particles are detected with an angular resolution of $\Delta \alpha \approx 1.1^{\circ}$ and a constant momentum resolution $\Delta p / p=2 \%$. These resolutions are the main parameters of the Gaussian smearing applied to the generated 3-momenta. After smearing, particle energies were recalculated in order to conserve the particle masses. The detection and identification probability was taken as $80 \%$ within the pseudo-rapidity range $|\eta|<0.7$ and for transverse momenta $p_{T}>150 \mathrm{MeV} / c$ and was considered to vanish outside of this range.

\section{Particle identification with PHOS}

In this and next sections, prompt photon identification and suppression of contamination due to

$\overline{3 a \oplus b}=\sqrt{a^{2}+b^{2}}$ 
hard $\pi^{0}$ are discussed. The identified prompt photon spectrum expected to be measured during one running period is presented.

Two different procedures to select prompt photons were applied: the Shower Shape Analysis (SSA), and the Isolation Cut Method (ICM). The former identifies photons by analyzing the shape of the shower in PHOS, and the latter tags and identifies a photon as prompt if it appears isolated, i. e., without charged particles in its vicinity. The ICM method is described in Section 5 .

\subsection{Shower shape analysis}

Photon spectra of $\gamma$-jet and jet-jet events were identified based mainly on the shape of the shower developped in the calorimeter and using Principal Component Analysis method (Ref. [13, 14]). With this method photons are identified with 3 kind of purities, high, medium and low, depending on the rejection quality of the heavy-ion environment. As example, we show the results for medium purity-photons: the prompt-photon identification efficiency was about $85 \%$ for $p p$ collisions and about $75 \%$ for $\mathrm{Pb}-\mathrm{Pb}$ collisions. The wrong identification probability, i. e., the probability of identifying a background event as a prompt photon, ranged from 0 to $40 \%$ in the case of onecluster $\pi^{0}$ and medium purity identification and from 5 to $15 \%$ in the case of hadrons. The remaining $\pi^{0}$ background from jet-jet events was still too high (Fig. 4). Further improvement of background suppression by increasing the photon purity is still possible but at the cost of unacceptable low photon identification efficiency. Thus, additional identification procedures had to be developed to overcome the limitations of the SSA method.

\section{The Isolation Cut Method}

The main idea behind the ICM is that prompt photons and partons produced in parton collisions are emitted in opposite directions. As there is no hadron from the parton jet flying in the same direction as the photon, the latter should appear isolated. However, the underlying event, in particu-
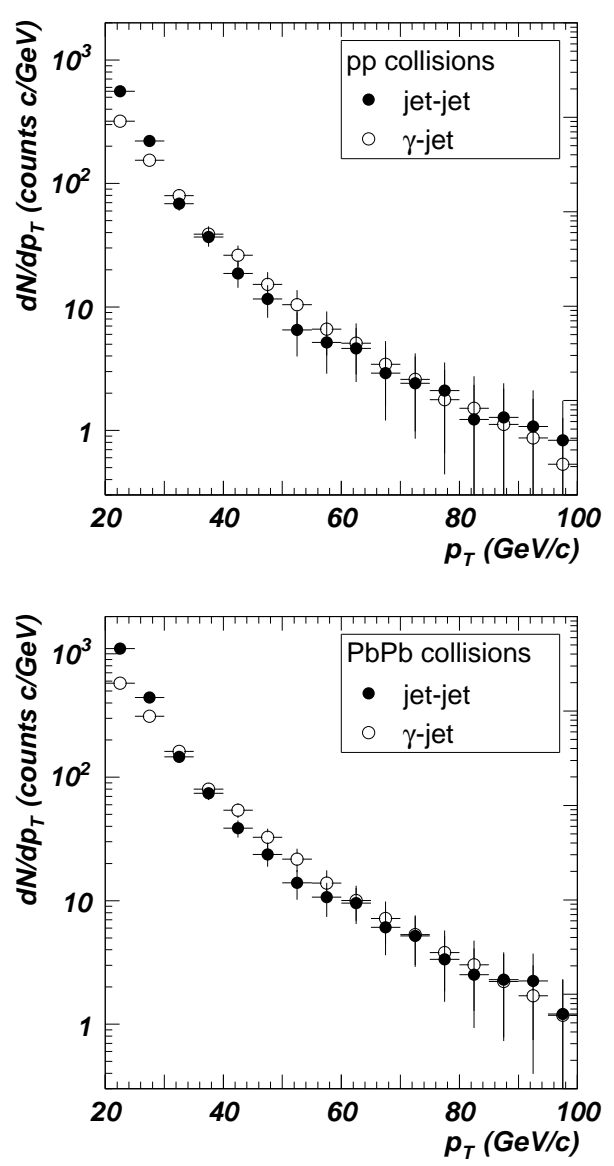

Fig. 4. Identified medium purity-photon spectra in jet-jet $(\bullet)$ and $\gamma$-jet events (०) with shower shape analysis in $p p$ (up) and $\mathrm{Pb}-\mathrm{Pb}$ (down) collisions at $5.5 \mathrm{~A} \mathrm{TeV}$ obtained for the expected luminosity in a standard LHC running year and with the corresponding statistical errors.

lar the one generated by the heavy-ion collision, may perturb this ideal topology. To overcome this difficulty, we developed two different isolation cut methods. Both methods search for hadrons inside a cone centered around the direction $\left(\eta_{0}, \phi_{0}\right)$ of high- $p_{T}$ photon candidates $\left(p_{T}>20 \mathrm{GeV} / c\right)$ identified by the SSA method. The cone is defined by the radius $R$ in the $(\phi, \eta)$ space,

$$
R=\sqrt{\left(\phi_{0}-\phi\right)^{2}+\left(\eta_{0}-\eta\right)^{2}} .
$$

The multiplicity inside the cone depends on the cone size and on the event type. For $\gamma$-jet events in $p p$ collisions, there is almost no particle inside the 
cone, independently of the energy of the prompt photon, but for jet-jet events there is a clear dependence of the multiplicity with the jet energy. Thus, applying $p_{T}$ cuts to the particles inside a cone around a photon candidate helps us to distinguish between $\gamma$-jet and jet-jet events. The two different ICM's decide if a photon candidate is isolated and should be tagged as a prompt photon as follows:

(i) No hadron with $p_{T}$ above a given threshold is found in the cone. We call this method standard ICM.

(ii) The sum of the transverse momentum of all hadrons inside the cone is smaller than a given threshold. We call this method ICMS (ICM with threshold on the Sum).

\subsection{Isolation cut method with particle $p_{T}$ threshold (ICM)}

We studied the ICM efficiency for cone sizes in the range $0.1<R<0.8$ and particle thresholds $p_{T}^{t h}=2,4$ and $6 \mathrm{GeV} / c$. We applied the ICM method to reconstructed events filtered through the TPC and PHOS acceptances and responses. The ICM efficiency was defined as the ratio of the number of isolated low purity-photons to the total number of low purity-photons ${ }^{4}$. The dependence of the ICM efficiency with the energy of the photon candidate for jet-jet events is shown in Figs. 5 and 6 for $R=0.1$ and 0.2 , respectively, and $p_{T}^{\text {th }}=2$, 4 and $6 \mathrm{GeV} / c$. The ratios of the integrals of the isolated low purity-photon spectrum and the total low purity-photon spectrum, for both $\gamma$-jet and jet-jet events and for different values of the cone size and $p_{T}^{t h}$, are reported in Table 4 .

The main features found are the following:

- $\gamma$-jet events: In the case of $p p$ collisions, the ICM efficiency is close to $100 \%$ independently of the cone size and of the $p_{T}$ threshold, but for $\mathrm{Pb}$ $\mathrm{Pb}$ collisions there is an important dependence of the efficiency on $R$ and $p_{T}^{t h}$; the smaller the cone size and the higher the $p_{T}$ threshold the better the isolation efficiency. No dependence of

4 The low purity-photon identification efficiency is about $90 \%$ for $p p$ collisions and about $80 \%$ for $\mathrm{Pb}-\mathrm{Pb}$ collisions.
Table 4

ICM integrated efficiency for $p p$ and $\mathrm{Pb}-\mathrm{Pb}$ collisions.

\begin{tabular}{cccc}
\hline$R$ & $p_{T}^{t h}=2 \mathrm{GeV} / c$ & $p_{T}^{t h}=4 \mathrm{GeV} / c$ & $p_{T}^{t h}=6 \mathrm{GeV} / c$ \\
\hline \hline & $p p$ collisions \\
\hline $0.1-0.8$ & $100 \%$ & $100 \%$ & $100 \%$ \\
\hline \multicolumn{5}{c}{ jet-jet events } \\
\hline 0.1 & $20 \%$ & $42 \%$ & $56 \%$ \\
0.2 & $16 \%$ & $41 \%$ & $56 \%$ \\
$0.3-0.8$ & $16 \%$ & $41 \%$ & $56 \%$ \\
\hline \multicolumn{5}{c}{ Pb-Pb collisions } \\
\hline 5 -jet events & & \\
\hline 0.1 & $80 \%$ & $98 \%$ & $99 \%$ \\
0.2 & $50 \%$ & $95 \%$ & $99 \%$ \\
$0.3-0.8$ & $34-8 \%$ & $93-84 \%$ & $98-97 \%$ \\
\hline \multicolumn{5}{c}{ jet-jet events } \\
\hline 0.1 & $15 \%$ & $39 \%$ & $56 \%$ \\
0.2 & $7 \%$ & $37 \%$ & $54 \%$ \\
$0.3-0.8$ & $4-1 \%$ & $36-31 \%$ & $54-53 \%$ \\
\hline
\end{tabular}

the isolation efficiency with the photon energy was observed.

- jet-jet events: The ICM gives a smaller probability of misidentifying a one-cluster $\pi^{0}$ as a photon in $\mathrm{Pb}-\mathrm{Pb}$ than in $p p$ collisions. The wrong identification probability increases with the $p_{T}$ threshold and is quasi independent of the cone size for large $p_{T}$ thresholds. The dependence of the wrong identification probability with the $p_{T}$ threshold is strong, ranging from about 50-60\% for $p_{T}^{t h}=6 \mathrm{GeV} / c$ to about $15-20 \%$ for $p_{T}^{t h}=$ $2 \mathrm{GeV} / c$ for $p p$ collisions and from about 50$60 \%$ for $p_{T}^{\text {th }}=6 \mathrm{GeV} / c$ to about $1-15 \%$ for $p_{T}^{t h}=2 \mathrm{GeV} / c$ for $\mathrm{Pb}-\mathrm{Pb}$ collisions.

The ratio of the $\gamma$-jet signal to the jet-jet background is reported in Table 5 and Figs. 7 and 8. In the case of $p p$ collisions, this ratio rises slightly with $R$ for $p_{T}^{t h}=2 \mathrm{GeV} / c$ but for $\mathrm{Pb}-\mathrm{Pb}$ collisions, this ratio shows a maximum at $R=0.3$, although at this value the ICM efficiency is almost the half than at $\mathrm{R}=0.2$ with only a moderate improvement of the signal to background ratio. For the largest $p_{T}$ thresholds $\left(p_{T}^{t h}=4\right.$ and $\left.6 \mathrm{GeV} / c\right)$, the signal to background ratio is smaller than for the lower 
$R=0.1$

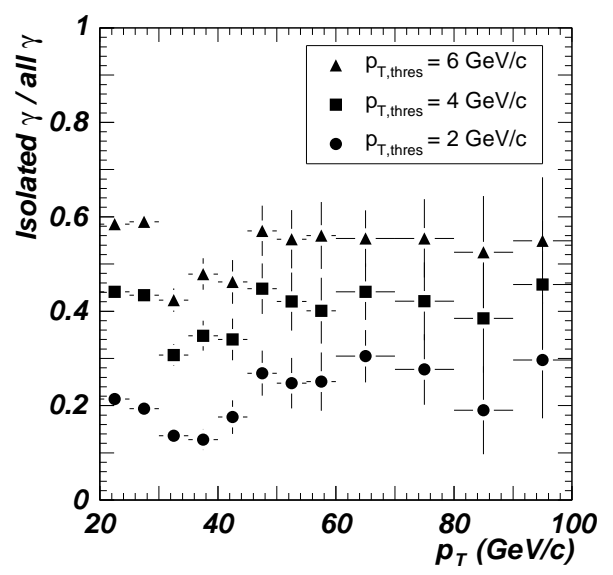

$R=0.2$

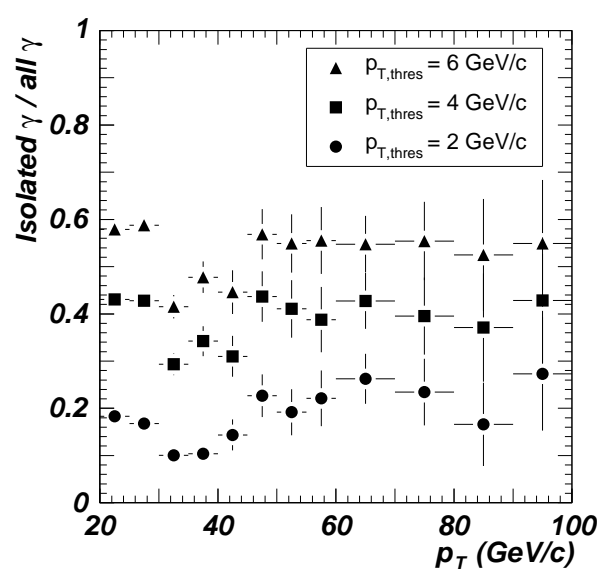

Fig. 5. Wrong Identification efficiency of ICM identified prompt photons as function of the photon $p_{T}$ for $R=0.1$ (up) and $R=0.2$ (down) cone sizes and 2, 4 and $6 \mathrm{GeV} / c$ $p_{T}$ thresholds for $p p$ collisions.

$p_{T}$ threshold $\left(p_{T}^{t h}=2 \mathrm{GeV} / c\right)$ and its dependence with $R$ is negligible. The optimum values of the ICM parameters, considering both signal to background ratio and efficiency, were found to be $R=$ 0.2 and $p_{T}^{t h}=2 \mathrm{GeV} / c$.
$R=0.1$

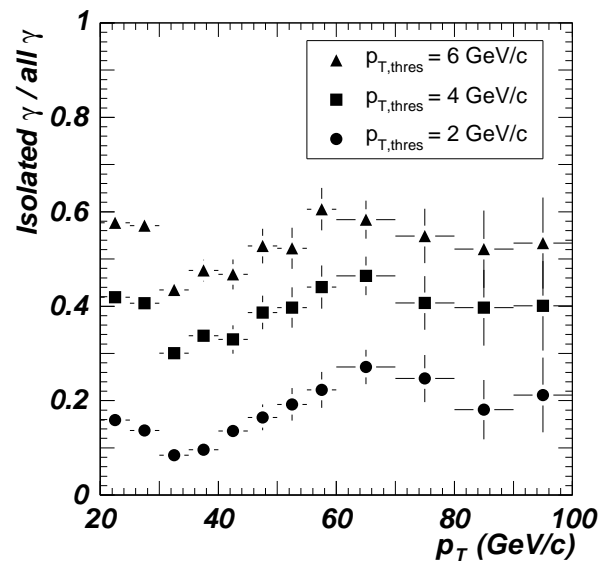

$R=0.2$

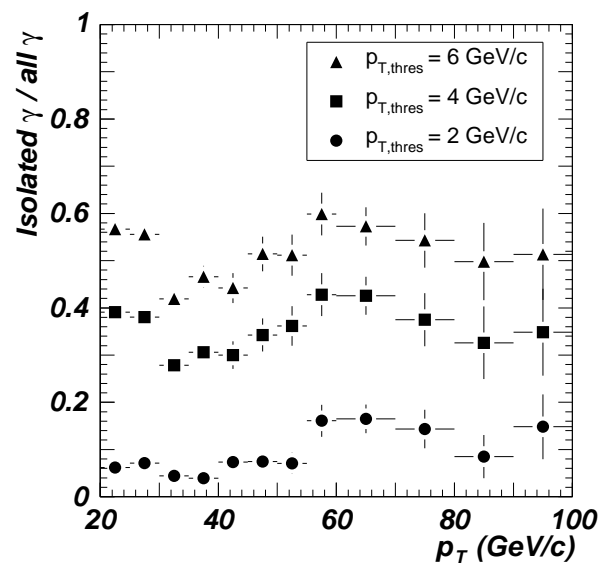

Fig. 6. Wrong Identification efficiency of ICM identified prompt photons as function of the photon $p_{T}$ for $R=0.1$ (up) and $R=0.2$ (down) cone sizes and 2, 4 and $6 \mathrm{GeV} / c$ $p_{T}$ thresholds $\mathrm{Pb}-\mathrm{Pb}$ collisions.

\subsection{Isolation cut method with threshold on the total $p_{T}$ sum (ICMS)}

The ICMS method sets a threshold $\Sigma_{p_{T}}$ to the sum of the transverse momentum of all the particles found in a given cone around the photon candidate. We studied the efficiency of the method for threshold values of $0.7,1,2$ and $3 \mathrm{GeV} / c$ for $p p$ collisions, and between 5 and $50 \mathrm{GeV} / c$ for $\mathrm{Pb}-\mathrm{Pb}$ collisions. It emerged that for $\mathrm{Pb}-\mathrm{Pb}$ collisions $\Sigma_{p_{T}}$ 
$R=0.1$

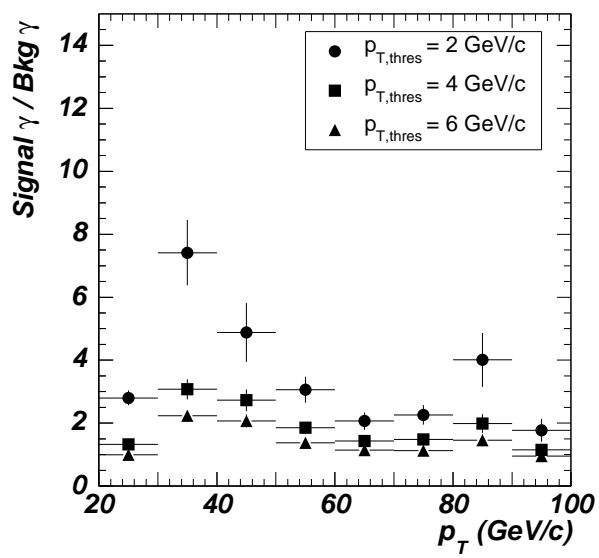

$R=0.2$

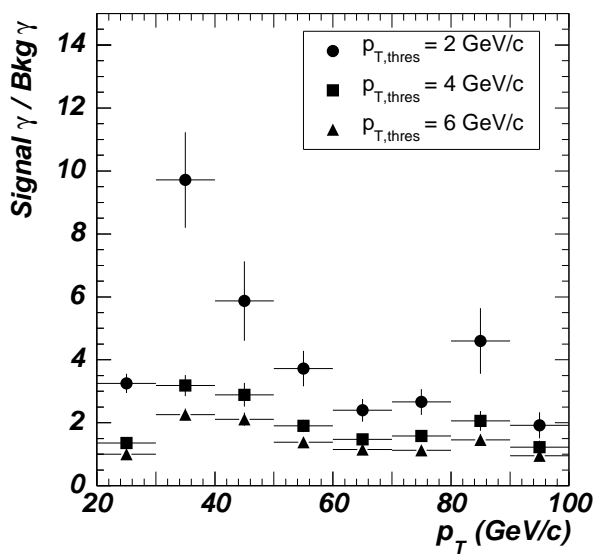

Fig. 7. Signal (identified prompt photons by SSA low purity and ICM from $\gamma$-jet events) to background (identified prompt photons by SSA low purity and ICM from jetjet events) as a function of $p_{T}$, for $R=0.1$ (up) and 0.2 (down), and $p_{T}^{\text {th }}$ of 2,4 and $6 \mathrm{GeV} / c$ and $p p$ collisions.

is not the appropriate parameter to compare results for different cone sizes due to its strong variation with $R$. Table 6 gives the mean and root mean square values of the particle distributions inside different cone sizes. We found that a more appropriate parameter is a threshold $\Sigma_{p_{T}}^{\prime}$ on the momentum sum density defined as

$$
\Sigma_{p_{T}}^{\prime}=\frac{\Sigma_{p_{T}}}{R^{2} \times f(R)},
$$

$$
R=0.1
$$

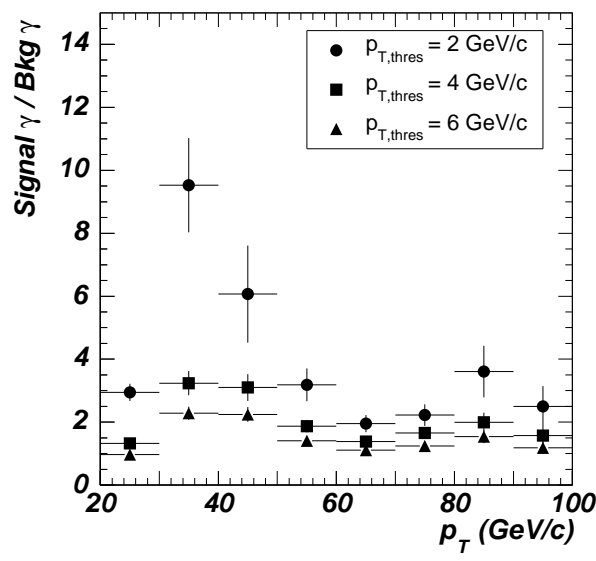

$R=0.2$

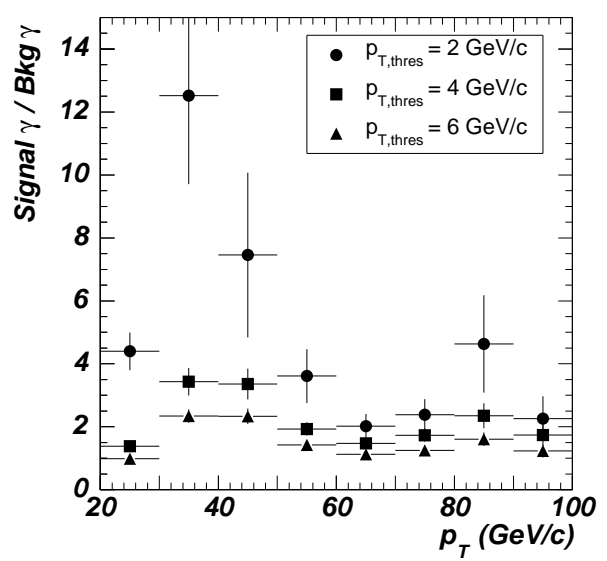

Fig. 8. Signal (identified prompt photons by SSA low purity and ICM from $\gamma$-jet events) to background (identified prompt photons by SSA low purity and ICM from jetjet events) as a function of $p_{T}$, for $R=0.1$ (up) and 0.2 (down), and $p_{T}^{t h}$ of 2,4 and $6 \mathrm{GeV} / c$ and $\mathrm{Pb}-\mathrm{Pb}$ collisions.

where $f(R)$ is a factor that takes into account the response function and acceptance of the various detectors involved. $f(R)$ is equal to one when detector response and acceptance are ignored. In our case, we took $f(R)=0.95-1.5 \times R$, deduced from fitting the mean values of the transverse momentum sum for detected events reported in Table 6 .

This method was applied to reconstructed events to calculate the ICMS efficiency for the different values of the cone size reported in Table 7 . 
Table 5

Integrated signal ( $\gamma$-jet) to integrated background (jet-jet) ratio obtained by the ICM method for $p p$ and $\mathrm{Pb}-\mathrm{Pb}$ collisions. $R$ and $p_{T}^{t h}$ are the cone size and particle momentum threshold.

\begin{tabular}{cccc}
\hline$R$ & $p_{T}^{t h}=2 \mathrm{GeV} / c$ & $p_{T}^{t h}=4 \mathrm{GeV} / c$ & $p_{T}^{t h}=6 \mathrm{GeV} / c$ \\
\hline \hline \multicolumn{3}{c}{$p p$ collisions } \\
0.1 & 3 & 1.4 & 1 \\
0.2 & 3.5 & 1.4 & 1 \\
$0.3-0.8$ & $3.6-3.7$ & 1.4 & 1 \\
\hline \multicolumn{5}{c}{$\mathrm{Pb}-\mathrm{Pb}$ collisions } \\
\hline 0.1 & 3 & 1.4 & 1 \\
0.2 & 4.2 & 1.4 & 1 \\
$0.3-0.8$ & $4.6-3$ & 1.5 & 1 \\
\hline
\end{tabular}

Table 6

Mean value and Root Mean Square (RMS) of the HIJING particle distributions of the transverse momentum sum as a function of the cone size for all the generated particles and only for detected particles.

\begin{tabular}{ccccc}
\hline & \multicolumn{1}{c}{$R=0.1$} & $R=0.2$ & $R=0.3$ \\
\hline \hline Generated & Mean $(\mathrm{GeV} / c)$ & 10 & 40 & 91 \\
& $\operatorname{RMS}(\mathrm{GeV} / c)$ & 3.8 & 9.8 & 18.9 \\
\hline Detected & Mean $(\mathrm{GeV} / c)$ & 9.2 & 27 & 43 \\
& $\operatorname{RMS}(\mathrm{GeV} / c)$ & 6.9 & 9.2 & 12 \\
\hline
\end{tabular}

The dependence of the ICMS wrong identification probability with the energy of the fake photon candidate for jet-jet events, for both $R=0.1$ and 0.2 , is shown in Figs. 9 and 10 for $p p$ and $\mathrm{Pb}-\mathrm{Pb}$ collisions, respectively. The following features are observed:

- $\gamma$-jet events: In the case of $p p$ collisions the ICMS efficiency is close to $100 \%$ for any threshold, independently of the cone size. On the other side, for $\mathrm{Pb}-\mathrm{Pb}$ collisions an important dependence on both $R$ and $\Sigma_{p_{T}}^{\prime}$ is found: the larger the cone size and $\Sigma_{p_{T}}^{\prime}$ the better the isolation efficiency. For example, for $\Sigma_{p_{T}}^{\prime}=1 \mathrm{TeV} / c$ the efficiency is about $50 \%$, increasing slightly with the cone size. No dependence of the ICMS efficiency with the photon energy was observed.

- jet-jet events: In $p p$ collisions, the ICMS probability of misidentifying one-cluster $\pi^{0}$ as photons decreases slightly with the cone size, and increases strongly with the $\Sigma_{p_{T}}^{\prime}$ threshold; for ex-
Table 7

Integrated ICMS efficiency for reconstructed $p p$ and $\mathrm{Pb}-\mathrm{Pb}$ events in PHOS and TPC.

\begin{tabular}{|c|c|c|c|}
\hline$\Sigma_{p_{T}}(\mathrm{GeV} / c)$ & $R=0.1$ & $R=0.2$ & $R=0.3$ \\
\hline \multicolumn{4}{|c|}{$\gamma$-jet } \\
\hline \multirow[t]{2}{*}{ any } & $100 \%$ & $100 \%$ & $100 \%$ \\
\hline & \multicolumn{3}{|l|}{ jet-jet } \\
\hline 0.7 & $6.1 \%$ & $3.2 \%$ & $2.1 \%$ \\
\hline 1.0 & $7.9 \%$ & $4.5 \%$ & $3.8 \%$ \\
\hline 2.0 & $15 \%$ & $10 \%$ & $8.6 \%$ \\
\hline \multirow[t]{2}{*}{3.0} & $22 \%$ & $16 \%$ & $15 \%$ \\
\hline & \multicolumn{2}{|c|}{$\mathrm{Pb}-\mathrm{Pb}$ collisions } & \\
\hline$\Sigma_{p_{T}}^{\prime}(\mathrm{TeV} / c)$ & $R=0.1$ & $R=0.2$ & $R=0.3$ \\
\hline \multicolumn{4}{|c|}{$\gamma$-jet events } \\
\hline 0.8 & $28 \%$ & $21 \%$ & $24 \%$ \\
\hline 0.9 & $36 \%$ & $34 \%$ & $36 \%$ \\
\hline 1.0 & $48 \%$ & $49 \%$ & $54 \%$ \\
\hline 1.1 & $56 \%$ & $62 \%$ & $73 \%$ \\
\hline \multicolumn{4}{|c|}{ jet-jet events } \\
\hline 0.8 & $7.2 \%$ & $9.1 \%$ & $14 \%$ \\
\hline 0.9 & $8.5 \%$ & $15 \%$ & $20 \%$ \\
\hline 1.0 & $12 \%$ & $20 \%$ & $28 \%$ \\
\hline 1.1 & $15 \%$ & $25 \%$ & $37 \%$ \\
\hline
\end{tabular}

ample, the probability is about $4-8 \%$ for $\Sigma_{p_{T}}=$ $1 \mathrm{GeV} / c$ and about $15-22 \%$ for $\Sigma_{p_{T}}=3 \mathrm{GeV} / c$ in the $0.1<R<0.3$ range. In the case of $\mathrm{Pb}-\mathrm{Pb}$ collisions, the ICMS wrong identification probability has an important dependence on both parameters: in the $0.1<R<0.3$ range, the wrong identification probability ranges between $12-28 \%$ for $\Sigma_{p_{T}}^{\prime}=1 \mathrm{TeV} / c$ but ranges between $15-37 \%$ for $\Sigma_{p_{T}}^{\prime}=1.1 \mathrm{TeV} / c$. The $\pi^{0}$ misididintification probability increases (i. e, the rejection decreases) with $\Sigma_{p_{T}}^{\prime}$. The ICMS probability of misidentifying a one-cluster $\pi^{0}$ as a photon is found to be almost independent of the $p_{T}$ of the photon candidate, as seen in Fig. 10.

The ratio of the $\gamma$-jet signal to jet-jet background is reported in Table 8 and Figs. 11 and 12. For $p p$ collisions, this ratio increases with $R$ and de- 
$R=0.1$

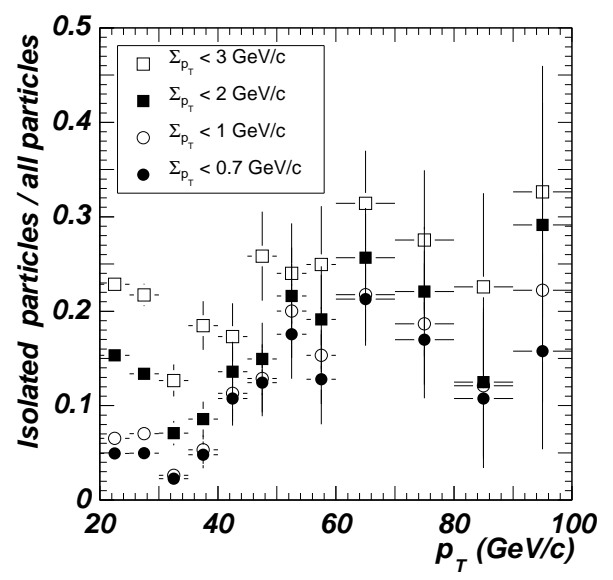

$R=0.2$

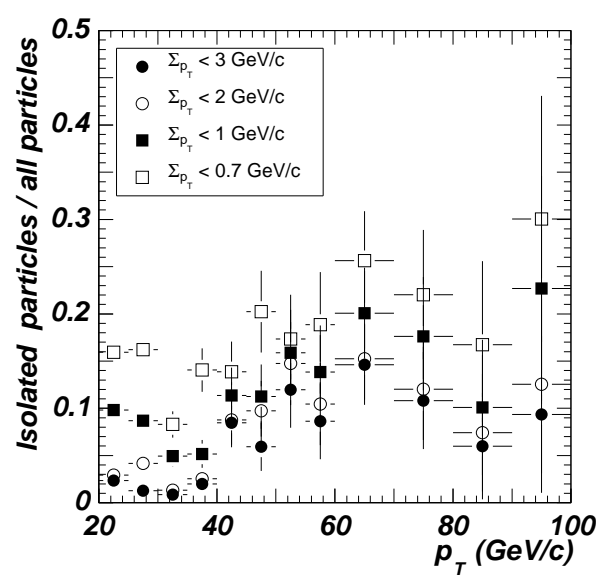

Fig. 9. Prompt photon wrong identification probability of ICMS as a function of the candidate $p_{T}$ for $R=0.1$ (up) and $R=0.2$ (down) and for different $\Sigma_{p_{T}}$ thresholds for $p p$ collisions.

creases with $\Sigma_{p_{T}}$. In the case of $\mathrm{Pb}-\mathrm{Pb}$ collisions, this ratio decreases with $R$ and shows a quite small dependence on $\Sigma_{p_{T}}^{\prime}$. The optimum values of the ICMS parameters were deduced from this pattern. For $p p$ collisions, the chosen parameters are $R=$ 0.2 and $\Sigma_{p_{T}}=0.7 \mathrm{GeV} / c$; although the signal to background ratio is somewhat better for $R=0.3$, we selected $R=0.2$ to compare with the ICM method. For $\mathrm{Pb}-\mathrm{Pb}$ collisions, we obtained the best signal to background ratio for $R=0.1$ and $\Sigma_{p_{T}}^{\prime}=$ $0.9 \mathrm{TeV} / c$.
$R=0.1$

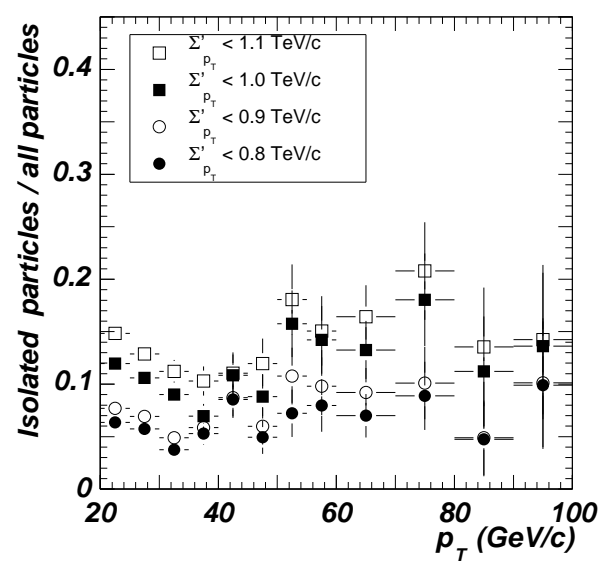

$R=0.2$

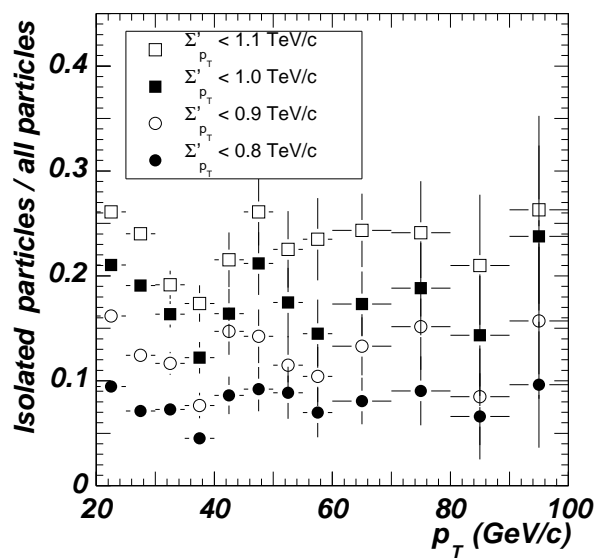

Fig. 10. Prompt photon wrong identification probability of ICMS as a function of the candidate $p_{T}$ for $R=0.1$ (up) and $R=0.2$ (down) and for different $\Sigma_{p_{T}}^{\prime}$ thresholds for $\mathrm{Pb}-\mathrm{Pb}$ collisions.

\subsection{Comparison of the ICM and ICMS methods}

The following conclusions are obtained from the comparison of the signal to background ratio given by the ICM and ICMS methods:

- In the case of $p p$ collisions, a threshold on $\Sigma_{p_{T}}$ is more efficient than a simple threshold on $p_{T}$. A $\gamma$-jet identification probability of $100 \%$ and a jet-jet wrong identification probability of $3 \%$ is obtained by the ICMS method with $R=0.2$ and $\Sigma_{p_{T}}=0.7 \mathrm{GeV} / c$ to be compared with a 


$$
R=0.1
$$

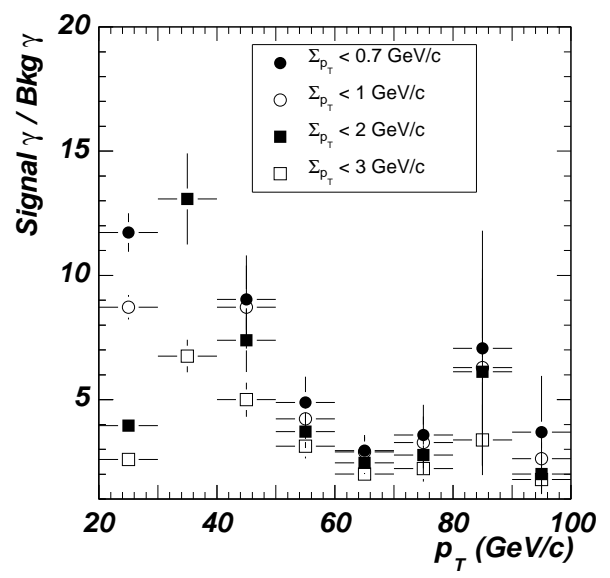

$$
R=0.2
$$

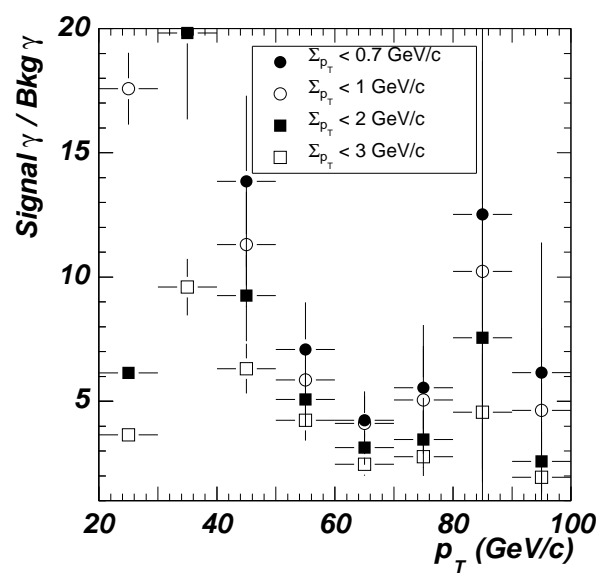

Fig. 11. Signal to background ratio as a function of $p_{T}$ for $R=0.1$ (up) and 0.2 (down), and for different $\Sigma_{p_{T}}$ thresholds for $p p$ collisions.

$\gamma$-jet identification probability of $100 \%$ and a jet-jet wrong identification probability of $16 \%$ obtained by the ICM with $R=0.2$ and $p_{T}^{t h}=$ $2 \mathrm{GeV} / c$.

- In the case of $\mathrm{Pb}-\mathrm{Pb}$ collisions, the ICM method achieves a better identification than the ICMS method. A $\gamma$-jet identification probability of $50 \%$ and a jet-jet wrong identification probability of $7 \%$ is obtained by the ICM with $R=0.2$ and $p_{T}^{t h}=2 \mathrm{GeV} / c$ to be compared with a $\gamma$-jet identification probability of $36 \%$ and a jet-jet

$$
R=0.1
$$

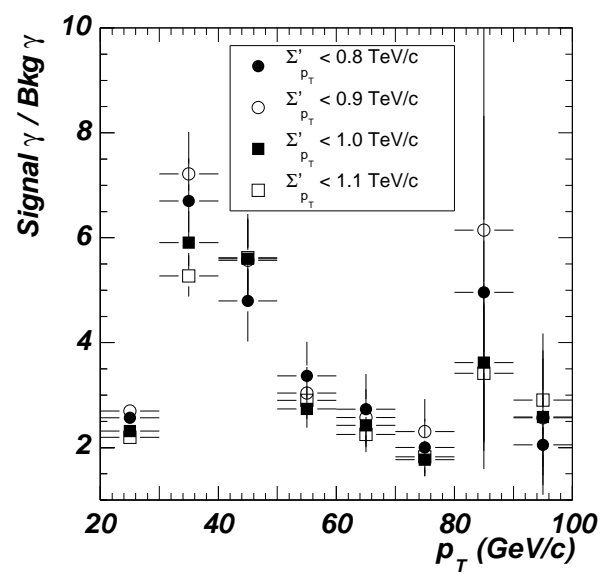

$$
R=0.2
$$

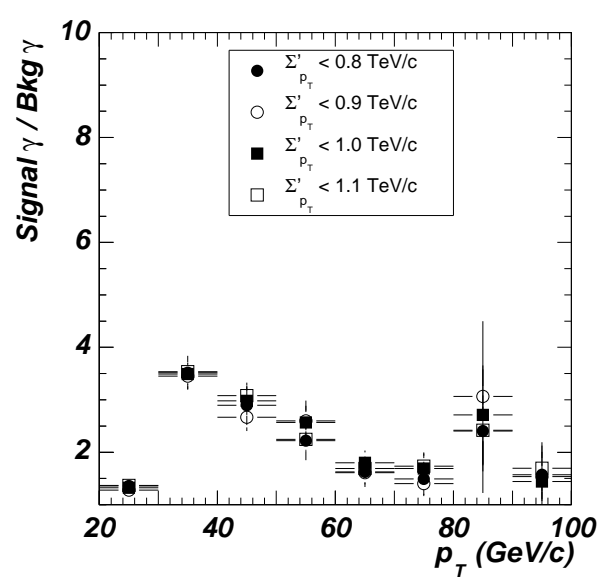

Fig. 12. Signal to background ratio as a function of $p_{T}$ for $R=0.1$ (up) and 0.2 (down), and for different $\Sigma_{p_{T}}^{\prime}$ thresholds for $\mathrm{Pb}-\mathrm{Pb}$ collisions.

wrong identification probability of $8.5 \%$ obtained by the ICMS method with $R=0.1$ and $\Sigma_{p_{T}}^{\prime}=0.9 \mathrm{TeV} / c$.

The identified prompt photon spectra obtained by the ICM and ICMS methods are presented in Fig. 13. The comparison with the spectra obtained by the SSA method in Fig. 4 clearly demonstrates a dramatic improvement of background rejection is achieved by ICMS for $p p$ collisions and by ICM for $\mathrm{Pb}-\mathrm{Pb}$ collisions.

At RHIC hadron spectra were observed to be quenched by a factor 5 at high $p_{T}[7-10]$. If the un- 
Table 8

$\gamma$-jet signal to jet-jet background ratio of the isolated photon integrated spectrum to the total photon integrated spectrum for reconstructed $p p$ and $\mathrm{Pb}-\mathrm{Pb}$ collisions in PHOS and TPC obtained by the ICMS method.

\begin{tabular}{cccc}
\hline$\Sigma_{p_{T}}(\mathrm{GeV} / c)$ & $R=0.1$ & $R=0.2$ & $R=0.3$ \\
\hline \hline \multicolumn{5}{c}{$p p$ collisions } \\
\hline 0.7 & 9.5 & 18 & 27 \\
1.0 & 7.4 & 13 & 15 \\
2.0 & 4.0 & 5.8 & 6.8 \\
3.0 & 2.6 & 3.6 & 4.0 \\
\hline \hline$\Sigma_{p_{T}}^{\prime}(\mathrm{TeV} / c)$ & $R=0.1$ & $R=0.2$ & $R=0.3$ \\
\hline \multicolumn{5}{c}{$\mathrm{Pb}-\mathrm{Pb}$ collisions } \\
\hline 0.8 & 2.3 & 1.3 & 0.99 \\
0.9 & 2.4 & 1.3 & 1.0 \\
1.0 & 2.2 & 1.4 & 1.1 \\
1.1 & 2.2 & 1.4 & 1.1 \\
\hline
\end{tabular}

derlying quenching mechanism still holds at LHC energies, the jet-jet contribution to the promptphoton background would be reduced by this factor, leading to a signal to background ratio of 20 instead of the 4.2 value given in Table 5 . The $\mathrm{Pb}$ $\mathrm{Pb}$ spectra displayed in Fig. 13 (ICM with $R=0.2$ and $p_{T}^{t h}=2 \mathrm{GeV} / c$ ) include this quenching factor.

\section{Prompt photon spectra}

The prompt photon spectra $N_{\gamma}^{i d}$ expected to be measured during one LHC running period, with their statistical and systematic errors, were calculated by adding the identified prompt photon spectrum from $\gamma$-jet events to the background spectrum from jet-jet events. From the identification efficiencies obtained by the simulations described above, we reconstructed the initial prompt-photon spectrum in the following way: let $N_{\gamma}$ be the original prompt photon spectrum, $N_{\pi^{o}}$ the original $\pi^{o}$ spectrum, $N_{h}$ the original hadron spectrum, $\varepsilon_{i}^{p i d}$ the identification probability of the particle $i$ as a photon by SSA and $\varepsilon_{i}^{i c m}$ the identification probability of particle $i$ as prompt photon by ICM, where $i$ stands for photon, one-cluster $\pi^{0}$ or any other hadron. We can write
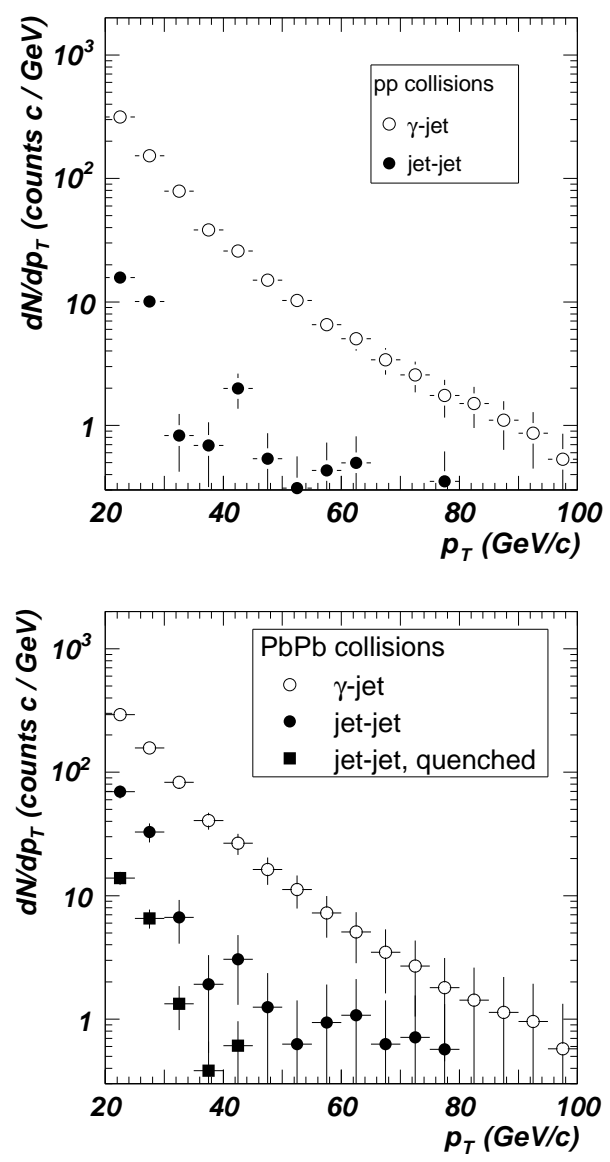

Fig. 13. Spectra of prompt photons identified in ALICE during a LHC running year with statistical errors by medium purity SSA and ICM methods for jet-jet $(\bullet)$ and $\gamma$-jet events (o) for $p p$ (up) and $\mathrm{Pb}-\mathrm{Pb}$ (down) collisions at $5.5 \mathrm{~A} \mathrm{TeV}$. A quenching factor of 5 for $\gamma$-like events is assumed for $\mathrm{Pb}-\mathrm{Pb}$ collisions ( $\mathbf{\square})$. ICMS was employed for $p p$ collisions and ICM for $\mathrm{Pb}-\mathrm{Pb}$ collisions.

$$
\begin{gathered}
N_{\gamma}^{i d}=N_{\gamma} \varepsilon_{\gamma}^{p i d} \varepsilon_{\gamma}^{i c m}+N_{\pi^{o}} \varepsilon_{\pi^{o}}^{p i d} \varepsilon_{\pi^{o}}^{i c m} \\
+N_{h} \varepsilon_{h}^{p i d} \varepsilon_{h}^{i c m},
\end{gathered}
$$

which can be rewritten as,

$$
\begin{gathered}
N_{\gamma}^{i d}=N_{\gamma}\left(\varepsilon_{\gamma}^{p i d} \varepsilon_{\gamma}^{i c m}+\frac{N_{\pi^{0}}}{N_{\gamma}} \varepsilon_{\pi^{o}}^{p i d} \varepsilon_{\pi^{o}}^{i c m}+\frac{N_{h}}{N_{\gamma}} \varepsilon_{h}^{p i d} \varepsilon_{h}^{i c m}\right) \\
=\zeta N_{\gamma} .
\end{gathered}
$$

Using the efficiencies obtained by our simulations, we deduced the various factors (the ICM 
wrong identification probabilities for $\pi^{\circ}$ and other hadrons were assumed to be equal) and the factor $\zeta$ for the different sets of identification criteria (PID photon classes, cone sizes and $p_{T}$ thresholds). The PID systematic error around the average value was calculated as the dispersion of the initial spectra obtained for each given set of identification criteria. This systematic error was added quadratically to the average background spectra in order to obtain the total systematic error. The statistical error was calculated from the photon statistics as $\sqrt{N_{\gamma}}$.

The final spectra of identified photons are compared to the original spectra for $p p$ and $\mathrm{Pb}-\mathrm{Pb}$ collisions (without quenching) in Figs. 14 and 15, respectively. In Fig. 16, the spectrum for $\mathrm{Pb}-\mathrm{Pb}$ collisions assuming a quenching factor of 5 , as observed at RHIC, is shown. The quenching main effect is a reduction of the systematic error by a factor 5 .

\section{Conclusions}

The isolation cut algorithms developed provide a tool to identify prompt photons produced in $p p$ and $\mathrm{Pb}-\mathrm{Pb}$ collisions in the ALICE experiment. These algorithms, when used simultaneously with shower shape analyses, allow to identify prompt photons in PHOS efficiently with excellent hadron and background rejection. Isolation cut methods are particularly efficient to reject decay and bremsstrahlung photons. The calculation through simulations of the different efficiencies involved in the detection process allows us to estimate the identified prompt photon spectrum to be measured by ALICE during a standard LHC running year with its statistical and systematic errors. This spectrum is a crucial observable to reveal the formation and properties of the quark-gluon plasma.

\section{Acknowledgment}

This work has been supported in part by the Spanish DGICYT under contract FPA2003-07581C02-01. One of us (G. C.) thanks Ministerio de Educación y Ciencia for a $\mathrm{PhD}$ fellowship under contract FP200-5452 and the "Training Site"
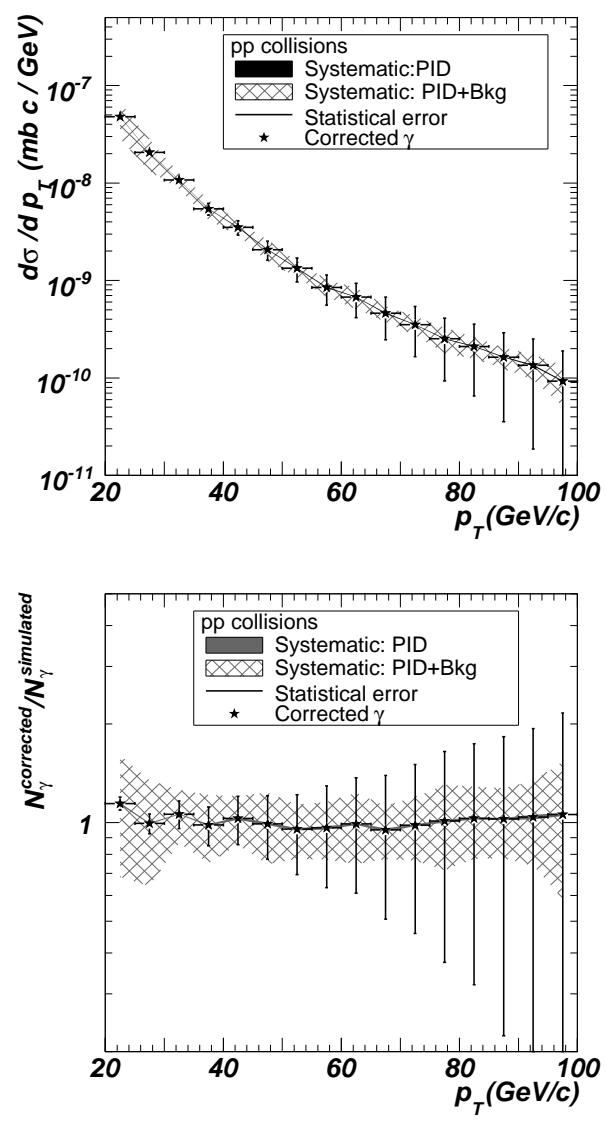

Fig. 14. Upper frame: Simulation of the prompt photon spectrum for $p p$ collisions at $5.5 \mathrm{TeV}$ incident energy measured in ALICE during a LHC running year with statistical and systematic errors. Lower frame: Ratio of the initial prompt photon spectrum obtained from the measured spectrum to the original simulated initial spectrum.

Marie Curie EU program under contract HPMTCT-2001-00346. We thank also the support of the INTAS grants 03-52-5747 and 05-103-7484.

\section{References}

[1] Hard Probes in Heavy Ion Collisions at the LHC. Ed. by M.L.Mangano, H.Satz, U.A.Wiedemann. Chapter 2, pp.123-246. ISBN: 92-9083-234-7. A.Accardi, et al. CERN-2004-009-B, Oct 2004; e-Print Archive: hep$\mathrm{ph} / 0310274$.

[2] J.D. Bjorken, FERMILAB-PUB-82-059-THY (1982).

[3] K. Adcox, et al., Phys. Lett. B 56 (2003), pp. 82-92. 

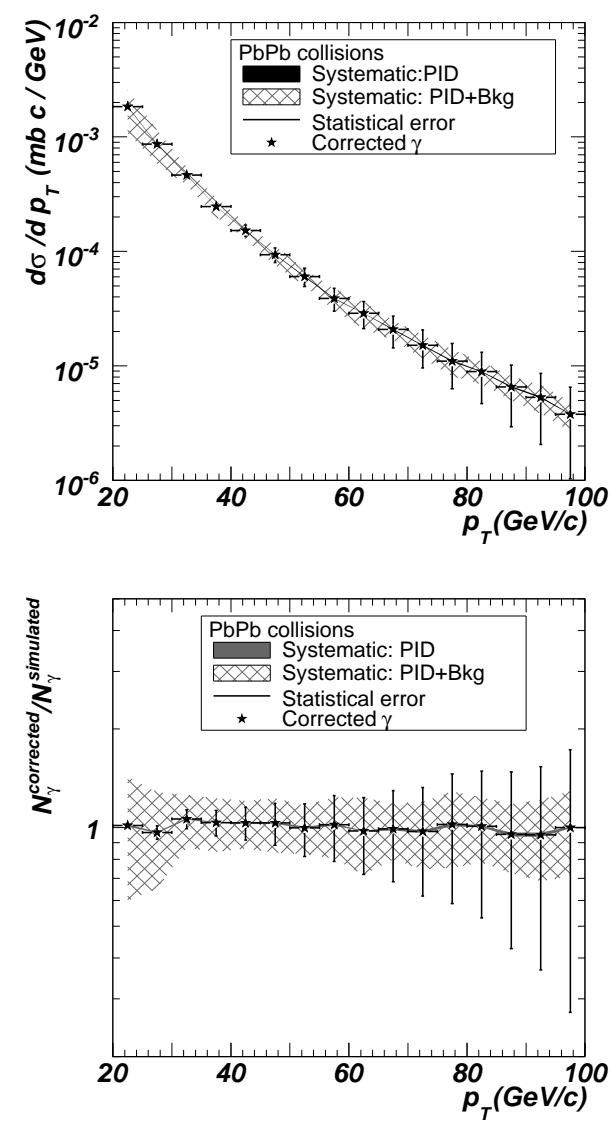

Fig. 15. Upper frame: Simulation of the measured prompt photon spectrum in ALICE during a LHC running year for $\mathrm{Pb}-\mathrm{Pb}$ collisions with statistical and systematic errors. Lower frame: Ratio of the initial prompt photon spectrum deduced from the measured spectrum to the original simulated spectrum.

[4] K. Adcox, et al., Phys. Rev. Lett. 88 (2002), p. 022301.

[5] C. Adler, et al., Phys. Rev. Lett. 89 (2002), p. 0202301.

[6] S. Adler, et al., Phys. Rev. Lett. 91 (2003), p. 072301.

[7] K. Adcox, et al., PHENIX Collaboration, Nucl. Phys. A 757 (2005), pp. 184-283.

[8] J. Adams, et al., STAR Collaboration, Nucl. Phys. A 757 (2005), pp. 102-183.

[9] I. Arsene, et al., BRAHMS Collaboration, Nucl. Phys. A $\mathbf{7 5 7}$ (2005), pp. 1-27.

[10] B.B. Back, et al., PHOBOS Collaboration, Nucl. Phys. A 757 (2005), pp. 28-101.

[11] C. A. Salgado and U.A. Wiedemann, Phys. Rev. Lett. 93 (2004), p. 42301.
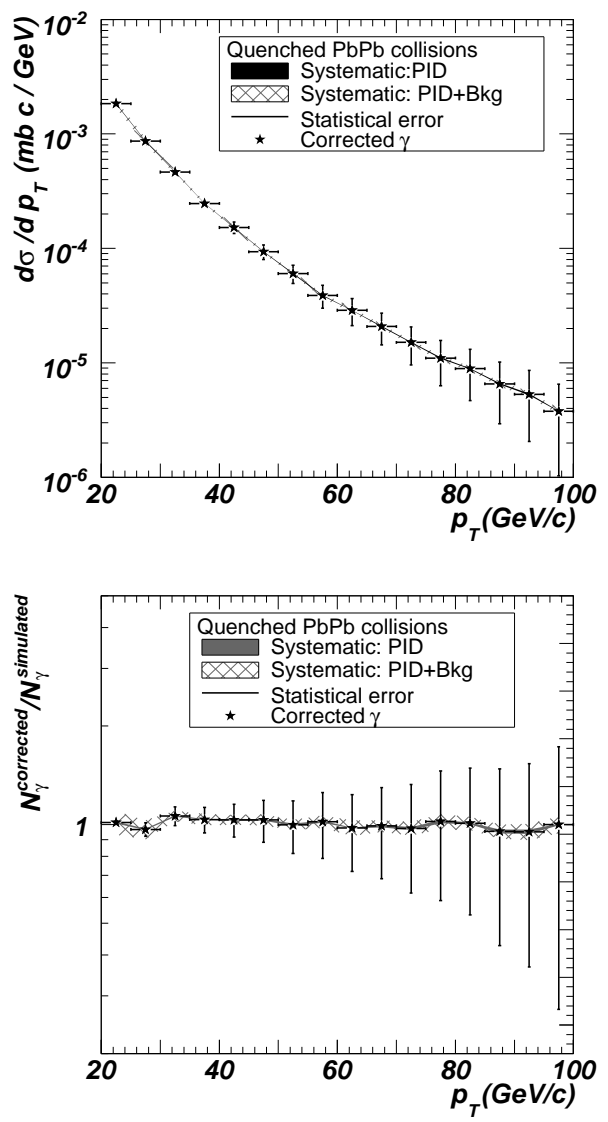

Fig. 16. Upper frame: Simulation of the measured prompt photon spectrum in ALICE during a LHC running year for quenched $\mathrm{Pb}-\mathrm{Pb}$ collisions with statistical and systematic errors. Lower frame: Ratio of the initial prompt photon spectrum deduced from the simulated spectrum to the original simulated initial spectrum.

[12] ALICE collaboration. Photon Spectrometer PHOS, Technical Design Report. CERN-LHCC-99-4, 5 March 1999.

[13] ALICE Collaboration, J. Phys. G.:Nucl. Part. Phys. 32 (2006), pp. 1295-2040

[14] G. Conesa, et al., Nuc. Ins. Met. A 537 (2005), pp. 363-367.

[15] T. Cormier, et al., ALICE Collaboration, The Electromagnetic Calorimeter Addendum to the ALICE Technical Proposal, CERN-LHCC-2006-014, CERNLHCC-96-32-ADD-3, 02 March 2006

[16] T. Cormier, et al., Eur. Phys. J. C 34 (2004), pp. s1.333-s1.345. 
[17] G. Conesa, et al., to be submmitted to Nucl. Instr. and Meth. Nucl. Res. A.

[18] ALICE Collaboration, J. Phys. G: Nucl. Part. Phys. 30 (2004), pp. 1517-1763.

[19] T. Sjostrand, et al., Comput. Phys. Commun. 135 (2001), pp. 238-259.

[20] T. Sjostrand, et al., PYTHIA 6.2: Physics and manual, hep-ph/0108264 (2001).

[21] M. Gluck, et al., Z. Phys. C 67 (1995), pp. 433-448.

[22] F. Arleo, et al., JHEP 11 (2004), p. 009.

[23] M. Gyulassy and X.N. Wang, Comput. Phys. Commun. 83 (1994), pp. 307-331.

[24] Hard Probes in Heavy Ion Collisions at the LHC. Ed. by M.L.Mangano, H.Satz, U.A.Wiedemann. Chapter 4, pp. 367-493. ISBN:92-9083-234-7. F.Arleo, et al., Photon physics in heavy-ion collisions at the LHC, Oct 2004. e-Print Archive: hep-ph/0311131.

[25] L. Aphecetche, et al., ALICE-INT-2002-02 (2002).

[26] D.V. Aleksandrov, et al., PHOS Collaboration, Nuc. Ins. Met. A A 550 (2005), pp. 169-184.

[27] ALICE collaboration. Technical Design Report of the Computing. CERN-LHCC-2005-018. 15 June 2005. AliRoot Web page: http://aliceinfo.cern.ch/Offline.

[28] ITS-ALICE collaboration, ALICE TDR 4 (1999).

[29] TPC-ALICE collaboration, ALICE TDR 7 (2000).

[30] M. Kowalski, ALICE-PR-2002-131 (2002). 